\title{
Dust Rotation and Swirl Morphology in Lunar Magnetic Anomalies
}

\author{
by \\ Michael Gerard
}

Thesis defense date

October 27th, 2017

Thesis advisor: Prof. Mihàly Horànyi

Department of Physics

Honors Council representative: Prof. Tobin Munsat

Department of Physics

Third reader: Prof. Andrew Hamilton

Department of Astrophysics 
Gerard, Michael

Dust Rotation and Swirl Morphology in Lunar Magnetic Anomalies Thesis directed by Prof. Mihàly Horànyi

The lunar surface is exposed to many violent interactions, which include the impinging solar wind, micrometeorite bombardment and large cometary nuclei. Yet in such a destructive environment, a few beautiful and enigmatic surface features called lunar swirls have emerged in the presence of crustal lunar magnetic anomalies (LMAs) [1]. Theories attempting to explain all the anomalous properties of lunar swirls range from impinging swarms of micrometeorites [2, 3], the magnetic shielding of solar wind [4, [5] and the electrostatic transport of lofted dust grains [6]. While all three of the theories seem to satisfy some of the swirl properties, none can sufficiently explain them all [7]. For this reason, additional or complementary models may be needed to resolve all observed phenomenon.

In this thesis, a new model is proposed which attempts to reconcile the solar wind standoff theory with the unique photometric properties of swirls. This model assumes that magnetized lunar dust, following a ballistic trajectory, will rotate in response to the torque they feel in the presence of an LMA, thereby producing a characteristic landing pattering in the swirl regolith.

To test the hypothesis, the rotation of needle like dust grains are simulated using a simple rotation model. The emergent landing pattern is then analyzed for regions of grain uniformity. No such model has previously been explored, and the results of the simulation seem to justify further consideration, especially since grain orientation may provide a novel approach to understanding the reflectance patterns observed from lunar swirls. 


\section{Dedication}

This thesis is dedicated to my parents, who have always shown me their continual love and support. Also, to my friends, who sat patiently through many conversations about moon dust. 


\section{Acknowledgements}

I would like to thank my advisor Mihàly Horànyi for his guidance and council throughout the entirity of this project, as well as his patience for my incessant inclusion of quaternions in nearly every conversation we had. I would also like to thank Jan Deca for his assistance with the implemnetation of the Reiner Gamma field data, and his willingness to help with numerous coding complications. I thank my thesis council for their dedication to the inclusion of undergraduates in scientific research, and everyone in the IMPACT lab who was a friend, collaborator and confidant. The road to my defense was paved with hard work, but the work was made easier thanks to all of you. 


\section{Contents}

\section{Chapter}

\begin{tabular}{lll}
\hline & Introduction & 1
\end{tabular}

1.1 What Are Lunar Swirls $\ldots \ldots \ldots \ldots \ldots \ldots \ldots$

1.2 Swirl Morphology $\ldots \ldots \ldots \ldots \ldots \ldots \ldots \ldots$

1.2 .1 Micrometeorite Swarms . . . . . . . . . . . . . . . . . 6

$1.2 .2 \quad$ Lunar Dust Transport $\ldots \ldots \ldots \ldots \ldots \ldots$

$1.2 .3 \quad$ Solar Wind Standoff $\ldots \ldots \ldots \ldots \ldots$

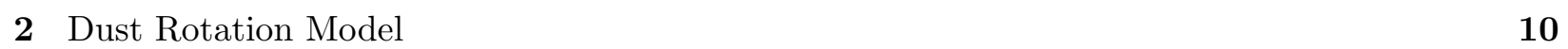

2.1 Introduction to the Model $\ldots \ldots \ldots \ldots \ldots$

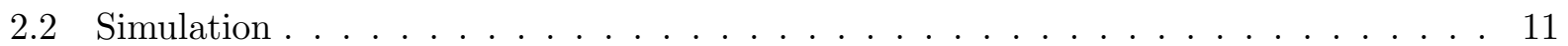

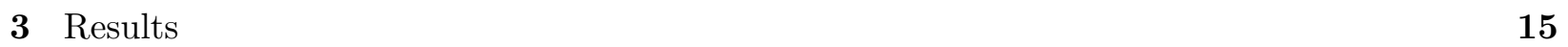

$3.1 \quad$ Single Particle in Dipole Field $\ldots \ldots \ldots \ldots$. . . . . . . . . . . . . . . 15

3.2 Many Particles in Dipole Field $\ldots \ldots \ldots \ldots$. . . . . . . . . . . . . 16

$3.3 \quad$ Ejected Particles in Reiner Gamma Field $\ldots \ldots \ldots$. . . . . . . . . . . . . . . . 18

3.4 Lofted Particles in Reiner Gamma Field . . . . . . . . . . . . . . . . . . . . . . . . . 20

$\begin{array}{|ll|}4 \text { Conclusion } & 24\end{array}$ 


\section{Appendix}

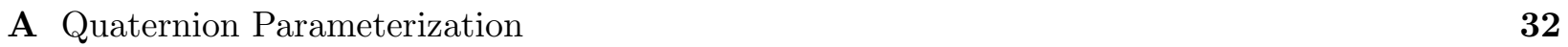

A.1 Introduction to Quaternions . . . . . . . . . . . . . . . . . . . 32

A.2 Application of the Quaternion Number Structure . . . . . . . . . . . . . . . . 34 


\section{Tables}

\section{Table}

2.1 Simulated Dust Parameters _. . . . . . . . . . . . . . . . . . . . . . 12 


\section{Figures}

\section{Figure}

1.1 Reiner Gamma swirl . . . . . . . . . . . . . . . . . . . . . . . . . . 2

$2.1 \quad$ Body frame and Space frame $\ldots \ldots \ldots \ldots \ldots$

3.1 These images show the simulated rotation of a single particle in a dipole field. The dipole lies $1 \mathrm{~cm}$ below the XY plane, with the magnetic moment pointing in the $+\mathrm{X}$

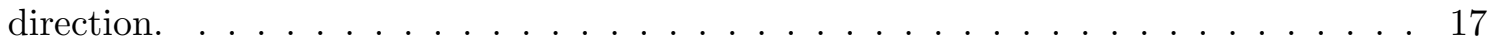

3.2 These images show the landing pattern from the dipole field simulation. The magnetic moment of the dipole lies 1cm behind the XY plane, pointing in the + Y direction. 19

3.3 Landing pattern from Reiner Gamma simulation with ejected dust grains. . . . . . . 21

3.4 Landing pattern from Reiner Gamma simulation with electrostatically lofted dust

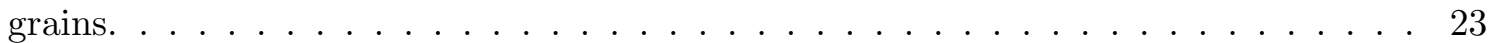




\section{Chapter 1}

\section{Introduction}

The intent of this chapter is to introduce the reader to the ongoing conversation about lunar swirl morphology. I will begin by describing the defining and anomalous characteristics of lunar swirls. Then, I will discuss the three most compelling theories for swirl morphology (1) micrometeorite swarms, (2) the electrostatic transport of lofted dust grains and (3) solar wind standoff. In my discussion of these three models, I will bring attention to their successes and shortcomings, providing a context for the exploration of this thesis into a new dust rotation model. With this model in mind, I will demonstrate a simple proof of concept in the subsequent chapters, thereby justifying future investigation.

\subsection{What Are Lunar Swirls}

Lunar swirls are one of the most striking features in the lunar regolith. They are identified by static patterns of highly reflective dust in the visible and near infrared spectrum [8]. Spanning nearly $3300 \mathrm{~km}^{2}$ across the western region of Oceanus Porcellarum [9], Reiner Gamma is the largest and most recognizable swirl on the moon, and it is shown in figure 1.1. Its anomalous regions of high reflectivity suggest an immature lunar regolith, which has, unlike the surrounding environment, not suffered the darkening effects of space weathering [6].

Perhaps the most curious feature of lunar swirls is their association with lunar magnetic anomalies (LMAs). While the moon has no global magnetic field, it does have multiple large-scale crustal magnetic fields, spanning hundreds of kilometers, with surface strengths as high as several 


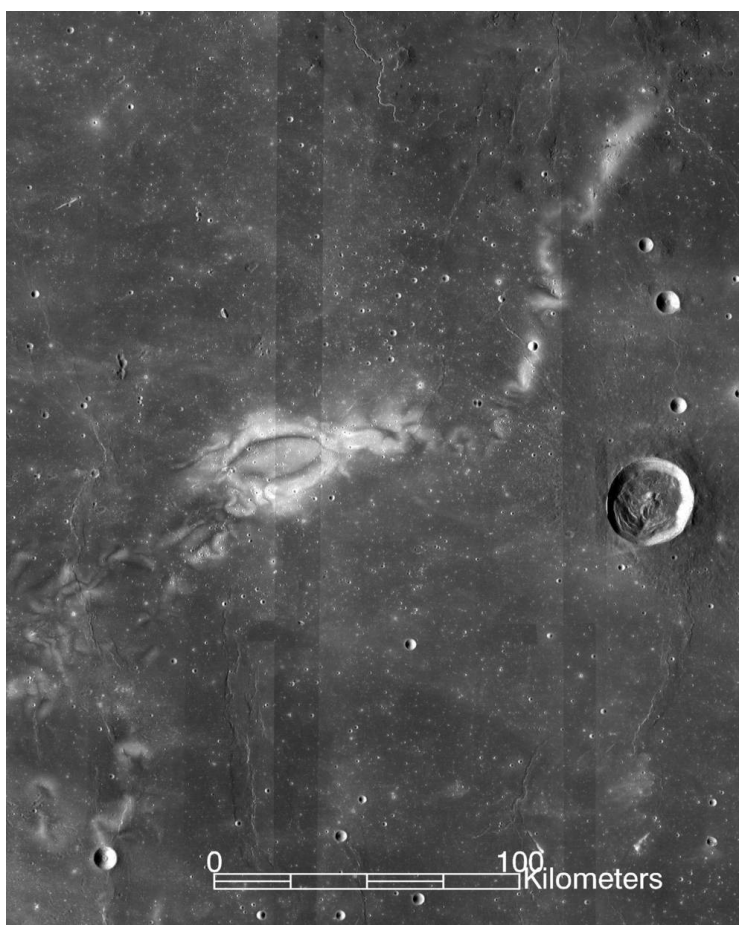

Figure 1.1: Reiner Gamma swirl

thousand nanoTeslas [10]. While not every LMA has a lunar swirl, every lunar swirl is found in an LMA [1]. For this reason, it is assumed that the formation of a lunar swirl is either dependent on the existence of an LMA, or will produce an LMA.

These swirls provide a natural laboratory in which to study the cross section between space weathering and crustal magnetization, providing a unique window to the moon's surface characteristics. The question of swirl morphology must therefore begin with insights into these two phenomenon. First, I will consider two theories concerning the formation of an LMA. Then, I will briefly discuss the process of space weathering. After that, I will describe the unique photometric properties of lunar swirls, which demonstrate an anomalous surface feature that is the primary motivation for the work herein.

The competing theories for the formation of an LMA generally fall into two categories; the slow formation that occurs in the presence of a former core dynamo, and the more rapid formation that results from the shock remanent magnetization of a high energy impact [10]. The former 
process is best supported in the case where an LMA is located at the center of an old (>3.85 Gyr) impact basin [11]. Numerical simulations have demonstrated that such impact events can raise the subsurface temperature to above 1,000 $\mathrm{K}$ for a period of $1 \mathrm{Myr}$ [10. These temperatures would exceed the curie point of metallic iron, thereby erasing any remanent magnetization in the regolith. Therefore, these LMAs must have been produced as the impact basin cooled in the presence of a long lived magnetic field, which would suggest a former core dynamo [11, [10].

Not all LMAs, however, occur in old impact basin. In fact, many LMAs have been correlated with the antipodal region of relatively young $(<3.85 \mathrm{Gyr})$ impact basin [5], [12]. It is believed that these impacts produce both seismic waves and a partially ionized ejecta cloud of vaporized particles, expanding thermally around the moon, converging in the region antipodal to the impact basin [13, [14]. The vapor cloud, reacting strongly with any ambient magnetic field, either embedded in the lunar surface or contained in the solar wind plasma, will substantially, and temporarily, increase the magnetic field strength in the antipodal region [13]. Then, either the seismic waves, the converging vapor cloud, or potentially both will produce surface pressures between 5-25 GPa [13. It has been shown experimentally that this pressure is sufficiently high to achieve shock remanent magnetization of the metallic iron particles found in the lunar regolith [15]. Therefore, one may expect the region antipodal to these young impact basin could adopt whatever magnetic field they encounter due to the converging ejecta cloud.

There are many other theories concerning the formation of an LMA, but to the author's knowledge, these two processes seem to be the most widely defended theories in the present literature. The process of space weathering, by comparison, appears to be more adequately constrained. It is a process that was first encountered during the Apollo missions, when it was observed that terrestrial rocks and minerals of composition comparable to those encountered on the moon, were lighter in color and higher in reflectance albedo than their lunar counterparts [16]. Over the last 50 years, the idea of space weathering has come to refer to the reddened reflectance spectrum, weaker absorption bands and lower albedo patterns observed in a material exposed to interplanetary environments [17, [18, 19]. 
On the moon, space weathering is generally understood to be the result of submicroscopic metallic iron (SMFe) particles, between 4 and $33 \mathrm{~nm}$ in diameter [20]. These SMFe particles coat the surface of larger particles [16], giving the lunar dust its unique optical properties as well as its ferromagnetic resonance [20], 21], 22], 223]. The production of these SMFe particles has two possible mechanisms; (1) the irradiation of the lunar surface by the solar wind, leading to the sputtering of metallic iron on to the surrounding particulates [16],224] and (2) the vapor deposition of metallic iron as a result of micrometeorite bombardment [25],[16], [26].

The darkening effects of hydrogen ion irradiation due to sputtering has been demonstrated on loose powders in many laboratory experiments [27], 28], [29], [30]. It has also been demonstrated with Ar ions [31, [32, 33], indicating the sputtering is responsible for the darkening effects rather than the ion species. Furthermore, many of these irradiation experiments have demonstrated darkening effects in conditions similar to $1 \mathrm{Myr}$ in a lunar environment [16], 34, [35].

The vapor deposition process has been simulated in laboratory environments by flash-heating of lunar rocks in nanosecond IR laser pulses [36], [37, [25], and in an electron beam furnace [26], all of which demonstrate the same darkening effects. The time scale of these laboratory experiments generally correspond to $100 \mathrm{Myr}$ of exposure to the current micrometeorite flux experienced on the moon [17], 38], describing a darkening process that occurs over a much longer period of time than ion irradiation. Therefore, any swirl formation mechanism must have occured within the last 1 to $100 \mathrm{Myr}$, or impede one or preferably both of these processes, leaving the swirl regolith with a high albedo and strong absorption bands.

There is, however, another characteristic of lunar swirls that is import when considering their formation, and that is their anomalous photometric properties compared to the lunar background [39], [40, [6]. Using X- and S-Band radar images taken from the Lunar Reconnaissance Orbiter, it has been shown that lunar swirls and the lunar background share the same radar backscattering and circular polarization ratios [41, indicating that lunar swirls have a smooth, reflective surface on the centimeter to decimeter scale similar to the surrounding environment [42, [43]. This is counter to the more general property found in the lunar regolith that young immature dust tends to be larger 
than darkened mature dust [44]. This peculiarity with swirls is similarly upheld with observations made from the European Space Agency's SMART-1 spacecraft [45, NASA's Clementine mission [46],47] and terrestrial based observations [48, all of which indicate an inverse relationship between lunar swirl albedo and phase function steepness.

If one assumes an isotropic surface structure with no preferred grain orientation, then theoretical models suggest a shallow phase function would be indicative of smooth surface characteristics at the millimeter scale [42, [43], [49]. This would imply an on swirl grain size equivalent to, or slightly larger than particles found in the off swirl background [50]. This, however, is in dramatic opposition to the high albedo dust found elsewhere on the moon. It is therefore an intent of this thesis to demonstrate a process in which the ferromagnetic properties of lunar dust may produce an anisotropy in the lunar regolith in the presence of an LMA, providing new insight into these unique photometric properties. To provide a better context for this later conversation, it is first necessary to discuss swirl morphology.

\section{$1.2 \quad$ Swirl Morphology}

Compiling the information, one develops a sense of the complexity in these swirl environments. They all occur in the presence of an anomalous magnetic field, demonstrate high albedo, weak absorption bands, and a reddened reflectance spectra, as well as unique photometric properties that indicate a reduced surface roughness when compared to similarly aged dust. Therefore, any swirl formation process must attempt to explain some, if not all of these anomalous characteristics. There are currently three such theories; (1) micrometeorite swarms which scour the lunar surface, revealing immature dust underneath [51, [2],[3], (2) the continual transport of young levitated dust into the electric fields produced by the interaction of the solar wind with an LMA [6] and (3) the deflection of the solar wind due to the local LMA, preventing space weather by ion irradiation [4],5]. In this section, I will consider the successes and shortcomings of each of these theories, making the case for an alternative or complementary model. 


\subsubsection{Micrometeorite Swarms}

The formation of micrometeorite swarms is uncertain. It has been proposed that a cometary nucleus will experience tidal disruption when passing through the Earth-moon system within 4 Earth radii of the Earth [9], though such encounters are not very common. It is also possible that a cometary nucleus may experience thermal breakup as it passes near the sun [50]. Either way, the dispersion of these meteorite swarms requires that the nucleus break up must occur near the Earth-moon system, or that the break up mechanism is gentle enough to produce small dense swarms [50]. Otherwise, the size of these swarms would quickly exceed the size of the moon. It is therefore assumed in swirl morphology that these meteorite swarms remain gravitationally bound until they collide with the lunar surface [50].

Upon collision, it is expected that these swarms will scour the lunar surface to depths of a few meters, uncovering young immature soil underneath [51, [2],[50]. The ejecta of these collisions then collide with one another, breaking into smaller fragments. These comminuted particles may then fall into the interstices that lie between the uncovered, coarsely grained dust [50], [52]. This mixing of comminuted older dust with the more coarsely grained young dust could explain the reduction in millimeter scale roughness observed on the lunar swirls [50, [47], [49], [52]. Another possible feature of this theory predicts that the ionized gaseous envelopes of the micrometeorite swarms would compress against the lunar surface, temporarily strengthening the local magnetic fields [53, [13]. Then, the energy of the swarm impact could produce sufficiently high pressures to acheive shock remnant magnetization of the metallic iron particles [13, [3], thereby producing the local LMA.

A swirl formed by a micrometeorite swarm would necessarily have occurred within the last 1 to $100 \mathrm{Myr}$, otherwise the uncovered dust would have been darkened in the space weathering process. Therefore, if the micrometeorite swarm theory is used to explain the correlation between swirls and LMAs, then these LMAs must be of an equivalently young age [2], 33. This is problematic when one considers the correlation between LMAs and impact basin dating back over 3.6 Gyr 
[54, [55],[56], suggesting an age for the LMAs many orders of magnitude larger than the age limit imposed by theory. So while micrometeorite swarms do provide a convenient explanation for the unique photometric properties of lunar swirls, they do not provide a similarly satisfying answer for their association with LMAs [6].

\subsubsection{Lunar Dust Transport}

The lunar dust transport model makes no qualms with LMAs dating back billions of years. In fact, the model is generally unconcerned with when or how an LMA was formed. Instead, the theory is based on the phenomenon of lunar dust lofting. Dust lofting may have been observed during the first Apollo missions, when astronauts reported seeing a distinct horizon glow during spaceship sunrise and sunset [59]. Some believe the glow may have been the result of forward scattered light off electrostatically lofted dust grains [60]. These grains, initially at rest on the lunar surface, are exposed to both UV radiation and the solar wind. This leads to a build up of electric charge due to photoelectric and/or secondary electron emission [61, 662]. The grains can then be electrostatically launched off the lunar surface, potentially reaching heights between a few centimeters [60] to over a kilometer [63], 64] above the surface.

This phenomenon may relate to LMAs through the appearance of steady state electric fields, which develop as a result of the charge separation due to the greater penetration depth of solar wind ions in the magnetic field compared to the solar wind electrons [65, [66], 667. Grains lofted off the surface will then be either attracted or repelled, depending on their charge, to the electrostatic field of an LMA. Based on rudimentary models, the time scale for the transport of these grains across a 2 to $10 \mathrm{~km}$ lunar swirl could be below the 1 Myr time scale for space weathering by ion irradiation [6]. Therefore, the model assumes that lunar swirls are geologically dynamic features

which have young immature dust continually transported in and out of the LMA in a time scale below that which is required for space weathering. Furthermore, the preferential lofting of small $(<45 \mu \mathrm{m})$ grains may explain the surface roughness characteristics associated with lunar swirls [6].

This theory may explain the association of lunar swirls with crustal magnetic fields as well 
as their unique photometric properties. This model, however, is the most recent addition to the field of swirl morphology. As such, the model is not well constrained in terms of lofting heights or flight times, both of which are explored over multiple orders of magnitude [6]. The model also predicts compositional differences between on and off swirl locations due to the preferred transport of feldspar material [6], but this prediction does not seem to be supported by the latest data from the Lunar Reconnaissance Orbiter [68].

\subsubsection{Solar Wind Standoff}

Similar to the dust transport model, solar wind standoff is not concerned with the origin of an LMA. Instead, the model supposes an independent process in which a nearby meteorite or cometary impact will deposit an ejecta cloud of unsurfaced young dust into a neighboring LMA [4],[5]. The immature dust, lying within the LMA, will then be shielded from the solar wind by the component of the LMA lying horizontal to the lunar surface [4],[57]. This shielding may be sufficient to reduce sputtering due to the solar wind, thereby slowing the space weathering process [5]. Micrometeorites would, of course, be unaffected by the LMA, so darkening affects may still take

place over $100 \mathrm{Myr}$, constraining the lifetime of a lunar swirl. But contrary to the micrometeorite swarm theory, this presents no contradiction with the formation of an LMA.

If true, solar wind standoff would nicely explain the association of lunar swirls with LMAs, but unfortunately, the shielding effects of LMAs have not been well demonstrated [6], [7]. In fact, the Reiner Gamma formation, which has the strongest LMA of all the known swirls [58], is the only swirl for which sufficient shielding has been modeled [5]. But rather then discrediting the theory, this shortcoming highlights the need for better flux measurements of the solar wind in regions of LMAs.

A more troubling limitation is that solar wind standoff does not easily explain the anomalous photometric properties observed on lunar swirls [39], 47], [40]. Therefore, even if solar wind standoff can be theoretically and experimentally verified, it would still be an incomplete theory on swirl morphology. In the next section, a complementary model to solar wind standoff will be presented. 
The intent of this model is to provide a potential new insight into the photometric properties of lunar swirls, thereby attempting to preserve solar wind standoff. 


\section{Chapter 2}

\section{Dust Rotation Model}

In the previous chapter, the topic of swirl morphology was introduced. The conversation was intended to demonstrate a need for a new or complementary model. In this chapter, one such model will be presented, and will be referred to as the dust rotation model. In the first section, I will describe its motivation, as well as the specific swirl characteristics it may one day help resolve. After that, I will describe the mathematical model used to simulate the relevant dust rotations.

\subsection{Introduction to the Model}

The fact that lunar dust has ferromagnetic properties has been understood since the first Apollo samples were returned from the moon [69], 223],[21]. What has not been explored, however, is how the dust will rotate in response to the torque it experiences in a magnetic field. Therefore, this dust rotation model aims to characterize the rotation of lunar dust as it follows a ballistic trajectory through an LMA. If lunar particles, originating from a nearby meteorite impact or an electrostatic lofting event, can be shown to rotate along their trajectory so as to align with a local magnetic field, then perhaps one could expect a grain orientation pattern to emerge in the vicinity of an LMA.

While such an orientation pattern may not produce the bright albedo features observed in a lunar swirl, it may affect the reflectance properties in a way that would produce the observed photometric anomalies. Therefore, if these anisotropic dust structures were to be confirmed, a dust

rotation model could play the role of a complementary theory to solar wind standoff. The desire 
to resolve solar wind standoff with the observed photometric properties comes from the fact that the theory seems to be the simplest explanation for the two most definitive characteristics of lunar swirls, their high albedo and association with LMAs.

It should be noted, however, that the effects of grain orientation on the reflectance properties of a particulate medium is not an established phenomenon. Therefore, regardless of any orientation patterns that may exist in the lunar regolith, many features of this model will have to be further developed before it is considered efficacious. But the work herein does provide an initial first step towards characterizing its viability.

\section{$2.2 \quad$ Simulation}

In this section, I will describe the model I used to simulate dust rotations. As a matter of notation, I will represent non unitary vectors with an arrow $(\vec{x})$, unitary vectors with a hat $(\hat{x})$ and time derivatives with Newtonian dot notation $(\dot{x})$.

The simulated dust grains are modeled as narrow cylinders, with a diameter one tenth their height $h$. Each dust grain has a characteristic diameter, which corresponds to the diameter of a spherical particle of equivalent volume. This characteristic diameter is constrained to a range of 0.1 to $10 \mu \mathrm{m}$, which is the diametric range of the most magnetic population of lunar dust [70]. The density of the dust grains is constrained as 2.3 to $3.2 \mathrm{~g} / \mathrm{cm}^{3}$, which represents an average density for a variety of lunar soil samples [71]. The magnetic moment for grains below $10 \mu \mathrm{m}$ in diameter

falls within the range of 0.62 to $1.2 \mathrm{Am}^{2} / \mathrm{kg}$ [70], and the electric charge on lofted dust grains of the same size can be estimated within $(1-5) \times 10^{4}$ units of elementary negative charge, in rough accordance with the patch charge model for lunar dust lofting [62]. The above ranges for our dust parameters are shown in Table 2.1.

The trajectory of each particle is modeled using Newton's Second Law, accounting for the gravitational and Lorentz forces.

$$
\ddot{\vec{r}}=-\frac{G M}{r^{2}} \hat{r}+\frac{Q}{m}(\dot{\vec{r}} \times \vec{B}(\vec{r})+\vec{E}(\vec{r}))
$$


Table 2.1: Simulated Dust Parameters

\begin{tabular}{lcc}
\hline Parameter & Min & Max \\
\hline Diameter $(\mu \mathrm{m})$ & 0.1 & 10 \\
\hline Density $\left(\mathrm{g} / \mathrm{cm}^{3}\right)$ & 2.3 & 3.2 \\
\hline Magnetic Moment $\left(\mathrm{Am}^{2} / \mathrm{kg}\right)$ & 0.62 & 1.2 \\
\hline Charge $\left(\mathrm{e}^{-}\right)$ & $1 \times 10^{4}$ & $5 \times 10^{4}$ \\
\hline
\end{tabular}

For simulations in which the dust grains are bound within a few meters of the lunar surface, the gravitational force is assumed to be constant. We can then replace the variable gravitational term $G M / r^{2}$ with $g=1.625 \mathrm{~m} / \mathrm{s}^{2}$.

To model grain rotation, each grain has its own body frame, represented by its principle axes $\hat{e}_{1}, \hat{e}_{2}$ and $\hat{e}_{3}$. The body frame is measured with respect to the space frame, represented with axes $\hat{x}, \hat{y}$ and $\hat{z}$ as shown in figure 2.1. In figure 2.1(b), the section on the northern hemisphere enclosed by the circle, bisected by $\hat{z}$, is the landing domain for any full scale lunar simulation. For the remainder of this thesis, any vector presented in the body frame will be denoted with a prime in its superscript, while any vector in the general space frame will be presented with no prime.

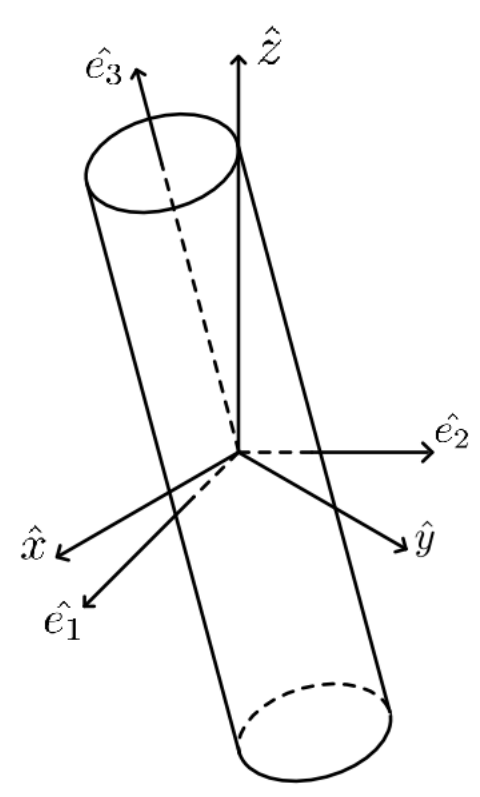

(a) Body frame

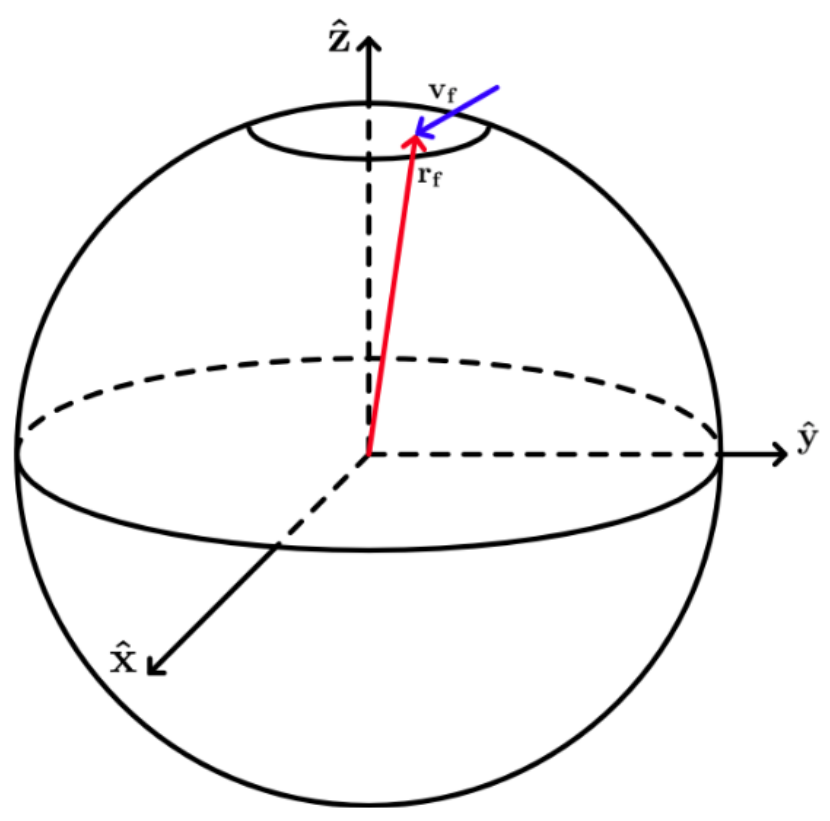

(b) Space frame

Figure 2.1: Body frame and Space frame 
The angular acceleration of each particle in its body frame is modeled using Euler's formula, and is shown in equation 2.2. The torque acting on the dust grain $\vec{N}$ is due to the interaction between the local magnetic field $\vec{B}$ and the grain's magnetic moment $\vec{\mu}$. The magnetic moment is assumed to be parallel with the grain's central principle axis $\vec{\mu}=\mu \hat{e}_{3}$.

$$
\begin{gathered}
\dot{\vec{\omega}}^{\prime}=\mathbf{I}^{-1}\left[\vec{N}^{\prime}-\left(\omega^{\prime} \times \mathbf{I} \vec{\omega}^{\prime}\right)\right] \\
\vec{N}^{\prime}=\vec{\mu}^{\prime} \times \vec{B}^{\prime}
\end{gathered}
$$

Since the grains are modeled as narrow, needle-like particles, their moment of inertia can be approximated with two non-zero eigenvalues.

$$
\mathbf{I} \approx \frac{1}{12} m h^{2}\left(\begin{array}{ccc}
1 & 0 & 0 \\
0 & 1 & 0 \\
0 & 0 & 0
\end{array}\right)
$$

The rotation of the grain is followed along its trajectory until the lowest part of the particle collides with the lunar surface. The collision is then modeled as an inelastic collision with a surface that has an infinite coefficient of friction. Therefore, we need not concern ourselves with any bouncing effects, and the lowest point of contact is treated as the new point of rotation. This changes the moment of inertia so that it has three non-zero eigenvalues.

$$
\mathbf{I} \approx m h^{2}\left(\begin{array}{ccc}
\frac{1}{3} & 0 & 0 \\
0 & \frac{1}{3} & 0 \\
0 & 0 & \frac{1}{4}
\end{array}\right)
$$

The torque acting on each grain must also be changed to include the forces due to gravity and the local electric field acting on the particle's center of mass. Since the collision takes place at the lunar surface, the variable acceleration due to gravity has been replaced by the constant $g$.

$$
\vec{N}=\hat{e}_{3} \times\left[\mu \vec{B}+\frac{1}{2} h \frac{\hat{e}_{3} \cdot \hat{r}}{\left|\hat{e}_{3} \cdot \hat{r}\right|}(Q \vec{E}-m g \hat{r})\right]
$$


After the collision, the grain's linear velocity $\dot{\vec{r}}$ contributes to its angular velocity $\vec{\omega}$ as shown in equation 2.7, where $\vec{\omega}_{o}$ represents the angular velocity immediately before the collision.

$$
\vec{\omega}=\vec{\omega}_{o}+\frac{2}{h} \frac{\hat{e}_{3} \cdot \hat{r}}{\left|\hat{e}_{3} \cdot \hat{r}\right|}\left[-\left(\dot{\vec{r}} \cdot \hat{e}_{2}\right) \hat{e}_{1}+\left(\dot{\vec{r}} \cdot \hat{e}_{1}\right) \hat{e}_{2}\right]
$$

Substituting the torque from equation 2.6 and the initial angular velocity from equation 2.7 into equation 2.2, the final rotations of the dust grain are tracked until $\left|\hat{e}_{3} \cdot \hat{r}\right|<0.1$. The final landing position of the particle's center of mass $\vec{r}$ is then recorded along with its orientation vector in the space frame $\hat{e}_{3}$ and the length of the grain's cylindrical height $h$.

It should be noted that to integrate equation 2.6, the angular velocity and particle orientation were parameterized using quaternions, which are preferred to Euler angles due to their numerical stability in highly rotationally dynamic systems [72]. Since this parameterization does not change the physical behavior of the system or make any additional assumptions, I will neglect to include the details here. If the reader is interested in a mathematical introduction to quaternions, refer to appendix A.1. If they would like a description of how I implemented the quaternion number structure, refer to appendix A.2. 


\section{Chapter 3}

\section{Results}

In this chapter, I will review the results of four different simulations. The first two simulations take place in a dipolar magnetic field, and are intended to verify the efficacy of the rotation model described in Chapter 2. The first simulation tracks the rotation of a single particle, while the second is used to construct a landing pattern from many simulated particles. The last two simulations both take place in the Reiner Gamma magnetic and electric field data, which is a collection of observational field strengths [73] and simulated field compression due to the interaction with the solar wind [74]. The first Reiner Gamma simulation will be for electrostatically lofted dust grains, while the second will be for grains ejected from the lunar surface due to a meteorite impact.

The code implemented in these simulations, and the subsequent analysis, was written by the author, using both numpy and scipy libraries. The differential equations were solved numerically using the VODE integrator in scipy's integrate library, which uses an implicit Adams method for non-stiff problems.

\subsection{Single Particle in Dipole Field}

As a first test for my simulation, I launched a single particle along a ballistic trajectory through a dipole field, with a downward acceleration of $9.8 \mathrm{~m} / \mathrm{s}^{2}$. The grain had a characteristic diameter of $10 \mu \mathrm{m}$, a mass of $2 \cdot 5 \cdot 10^{-11} \mathrm{~kg}$, and a magnetic moment of $3 \cdot 10^{-11} \mathrm{Am}^{2}$. The magnetic field is produced by a dipole placed at the center of the $X Y$ domain, $1 \mathrm{~cm}$ below the surface. The dipole's magnetic moment points in the positive $\mathrm{X}$ direction with a magnitude of $0.875 \mathrm{Am}^{2}$, which 
is roughly equivalent to a $1 \mathrm{~cm}^{3}$ neodymium magnet. In figure 3.1 I have shown the rotation of the particle along its trajectory, which begins near $\mathrm{Y}=0.5 \mathrm{~m}$ and lands near $\mathrm{X}=0.5 \mathrm{~m}$. The red end of the grain indicates the direction of its magnetic moment. The grain has no initial angular velocity, so any rotation is due to the torque it experiences as a result of the magnetic field. The initial and final scalar products between the particle's magnetic moment and the local magnetic field are approximately 0.27 and 0.97 , respectively, indicating the grain rotated to align with the magnetic field.

\subsection{Many Particles in Dipole Field}

The next verification was to simulate many grains being dropped into a magnetic field. The downward acceleration and magnetic field for this simulation were identical to those used for the single particle simulation, with the only exception being that now the magnetic moment of the dipole points in the positive $\mathrm{Y}$ direction. The grain parameters were randomized in accordance with table 2.1, except the grains were initialized with zero electric charge rather than the values listed in the table. Each grain was also initialized with zero angular velocity within a $0.75 \mathrm{~m}$ radial disk centered above the magnetic dipole at a height of $1 \mathrm{~m}$. Therefore, this simulation was an attempt to recreate the landing pattern that would be produced by dropping a collection of magnetic lunar dust onto a surface with a $1 \mathrm{~cm}^{3}$ neodymium magnet underneath it.

The results are shown in figure 3.2 . The figure was made by separating the landing region into 250,000 rectilinear bins. Within each bin, each grain's landing vector was summed together, weighted with respect to the grain's length. The resultant was then normalized to produce an average orientation for each bin. That data was used to produce a landing pattern which is superimposed on a local correlation background in figure 3.2(b), where a radially reduced subset of the data is shown so as to resolve the individual landing vectors represented by the black lines. The background, which is shown over the full domain in figure $3.2(\mathrm{a})$, was created by taking the average scalar product between the orientation vector in one bin and the corresponding vectors in the eight bins adjacent to it. Therefore, the plot will depict regions of uniform grain orientation in 


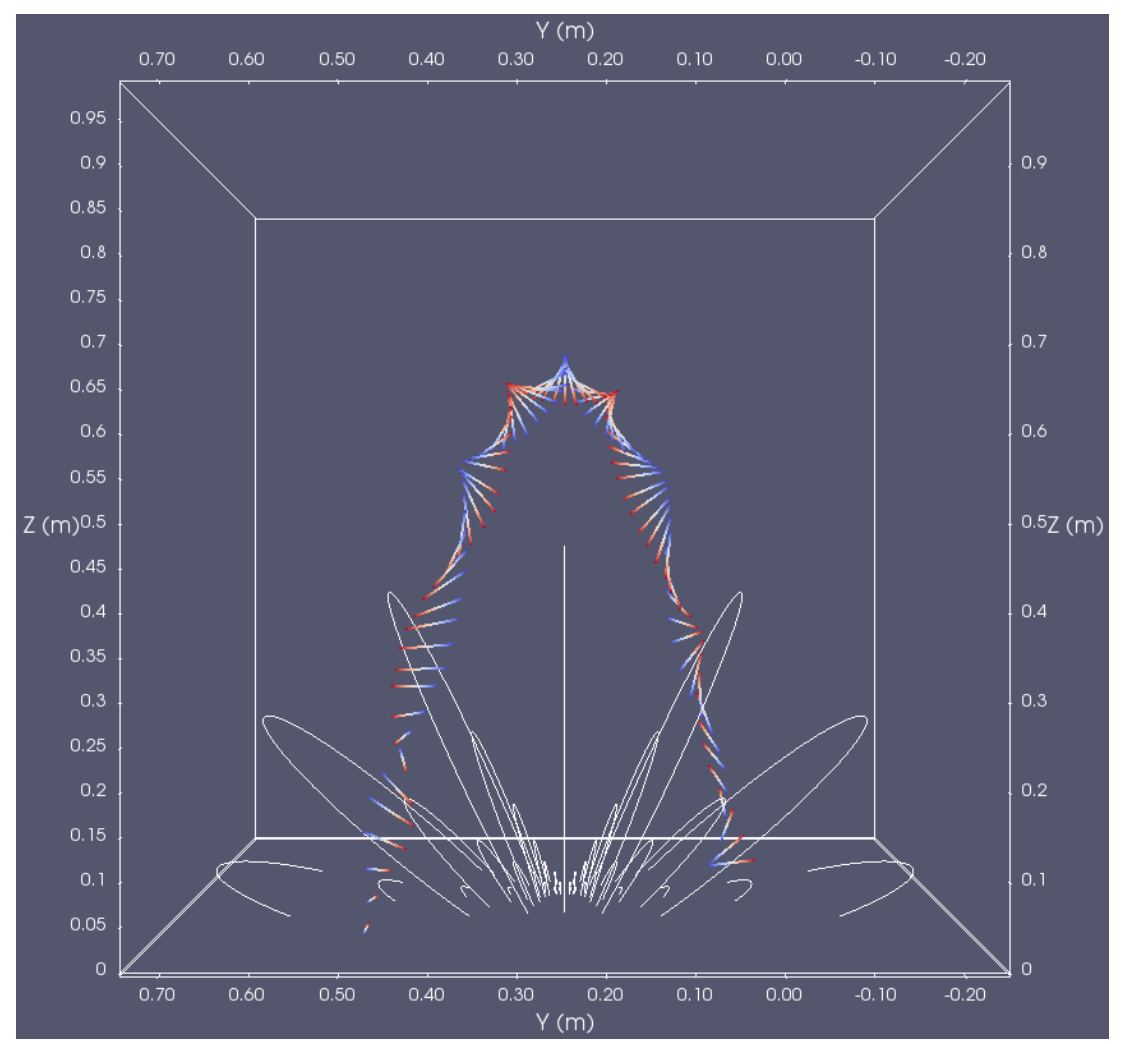

(a) Initial position: $Y \approx 0.5 \mathrm{~m}$

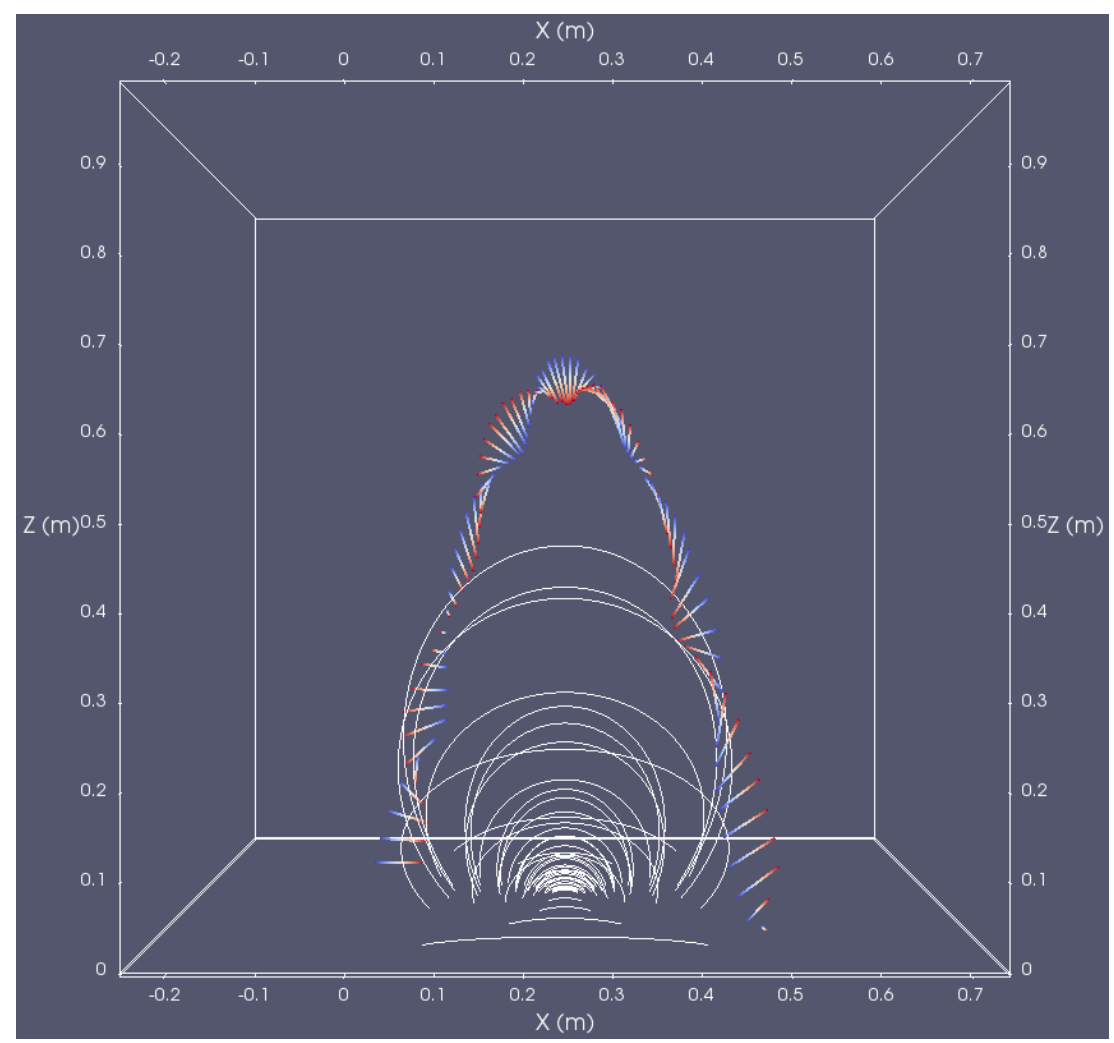

(b) Landing position: $X \approx 0.5 \mathrm{~m}$

Figure 3.1: These images show the simulated rotation of a single particle in a dipole field. The dipole lies $1 \mathrm{~cm}$ below the XY plane, with the magnetic moment pointing in the $+\mathrm{X}$ direction. 
red, and regions of random orientation shifted towards blue and violet.

The results of this simulation indicate significant grain alignment within the $0.75 \mathrm{~m}$ radial disk of the landing surface, thereby producing the iconic symmetric lobes one may expect from magnetic particles in a dipolar field. In addition to verifying the efficacy of the model, I also intend for these results to serve as a qualitative verification for the local correlation plot's ability to represent regions of grain uniformity. While the technique is surely not be a rigorous treatment of the data, it does seem to effectively highlight regions of uniformity, and will therefore be used in the analysis of all subsequent simulations.

\subsection{Ejected Particles in Reiner Gamma Field}

For the Reiner Gamma simulation, each particle was initialized with a collection of randomly sampled parameters in full accordance with table 2.1. For this initial simulation, the dust grains were assumed to originate as ejecta due to a nearby meteorite impact. To determine the initial conditions for such grains, I randomly sampled a point along the lunar surface within a $125 \mathrm{~km}$ radius of the Reiner Gamma swirl. That point was then used as an approximation for the dust grain's final landing position $\vec{r}_{f}$. The grain's final speed $\vec{v}_{f}$ was randomly sampled from a range of 250 to $1000 \mathrm{~m} / \mathrm{s}$, a range which constitutes nearly 75 percent of ejected grains according to the speed distribution functions derived from the LADEE/LDEX measurements [75]. The ejector angle was sampled from 5 to 20 degrees, measured off the normal to the lunar surface, and the azimuthal angle was between 0 and $2 \pi$ radians.

Due to the prohibitively long processing time of such a rotationally dynamic system, it seemed unreasonable to track the rotation of the ejected dust grains along their entire ballistic path. Instead, I used $\vec{r}_{f}$ and $\vec{v}_{f}$ to characterize each grain's orbital ellipse, assuming a gravitationally dominate trajectory. Then, I calculated the point of intersection between the orbital ellipse and a plane lying normal to the $\hat{z}$ axis of the space frame $1 \mathrm{~km}$ above the surface of the moon. The intersection between the ellipse and the plane, and the corresponding velocity at that point, were used for the initial position and velocity of the ejected dust grain. Therefore, each grain was 


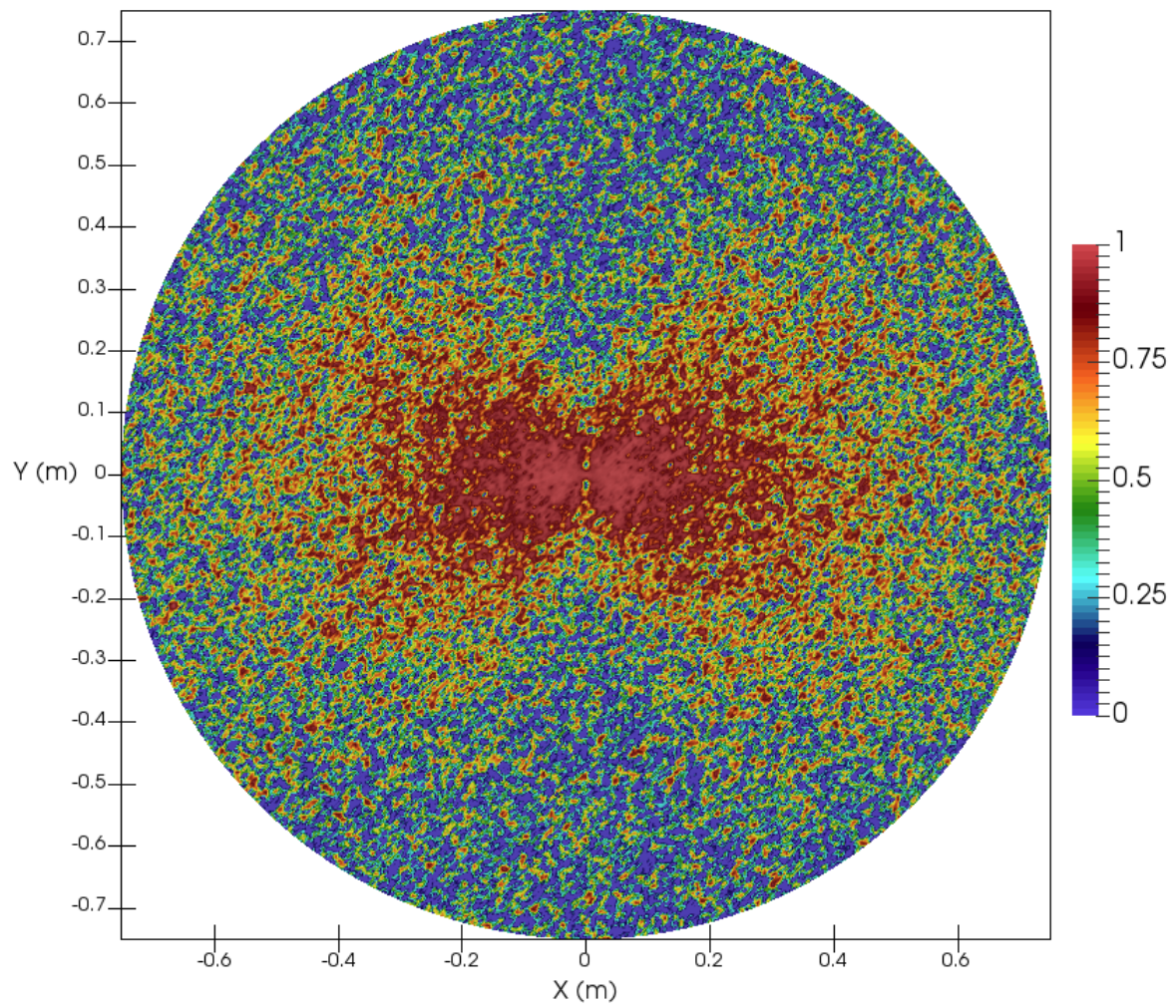

(a) Local correlation is shown over the full domain of the simulation.

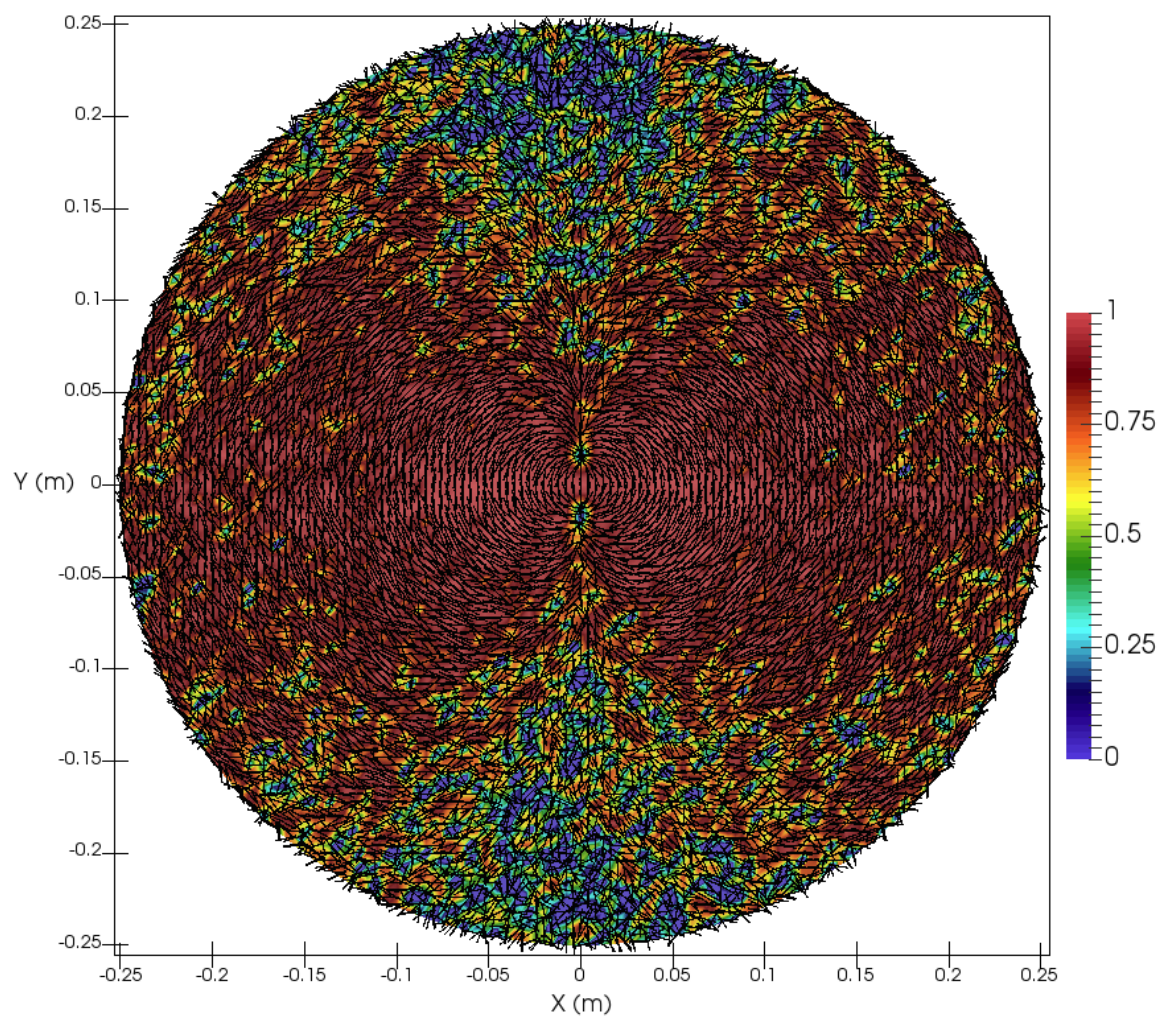

(b) The black lines represent the averaged landing vectors. The background is the radially reduced local correlation.

Figure 3.2: These images show the landing pattern from the dipole field simulation. The magnetic moment of the dipole lies $1 \mathrm{~cm}$ behind the $\mathrm{XY}$ plane, pointing in the $+\mathrm{Y}$ direction. 
initialized within the strongest region of the Reiner Gamma field data. Each dust grain was then given a random orientation and angular velocity. The later of which was determined from sampling a range of $4 \pi$ to $10 \pi \mathrm{rad} / \mathrm{s}$ along both azimuthal principle axes. Without any supporting data for the angular velocities of ejected lunar dust, this range was selected as a balance between a relatively high angular speed and a low processing time.

The simulation was allowed to run for over 2 million particles. The results are shown in figure 3.3. The landing pattern, produced in the same fashion as the landing pattern in section 3.2 , using 34,225 rectilinear bins, is represented by the black lines, which are superimposed over the local correlation plot. From this plot it is clear there is no significant region of grain uniformity. This is most likely due to the low rotational kinetic energy of the dust grains compared to their much higher linear kinetic energies. This energy discrepancy may obfuscate any potential orientation pattern due to the dominating affect of the grain's linear velocity $\dot{\vec{r}}$ in equation 2.7, where the rotation of the dust grain after its initial collision with the lunar surface will be almost entirely determined by its linear velocity. The truncated ballistic path may also contribute to the absence of any emergent pattern, though I suspect its effect would be negligible when compared to the difference in linear and rotational kinetic energies.

\subsection{Lofted Particles in Reiner Gamma Field}

In an attempt to reduce the discrepancy between the linear and rotational kinetic energies of the dust grains, I simulated the rotations of electrostatically lofted grains, which would have linear kinetic energies significantly lower than grains ejected from a meteorite impact. The dust parameters used were identical to those used in section 3.3 . In this simulation, however, the randomly sampled point along the lunar surface, within the $125 \mathrm{~km}$ of the Reiner Gamma swirl, was used as the initial position of each dust grain $\vec{r}_{i}$. The observed dust lofting on the lunar surface suggests lofting heights between 3 and $30 \mathrm{~cm}$ [60]. Therefore, with a randomly selected ejector angle between 0 and $\pi / 4$ radians, measured off the normal to the lunar surface, and an azimuthal angle between 0 and $2 \pi$ radians, the magnitude of each grain's initial velocity was constrained to a 


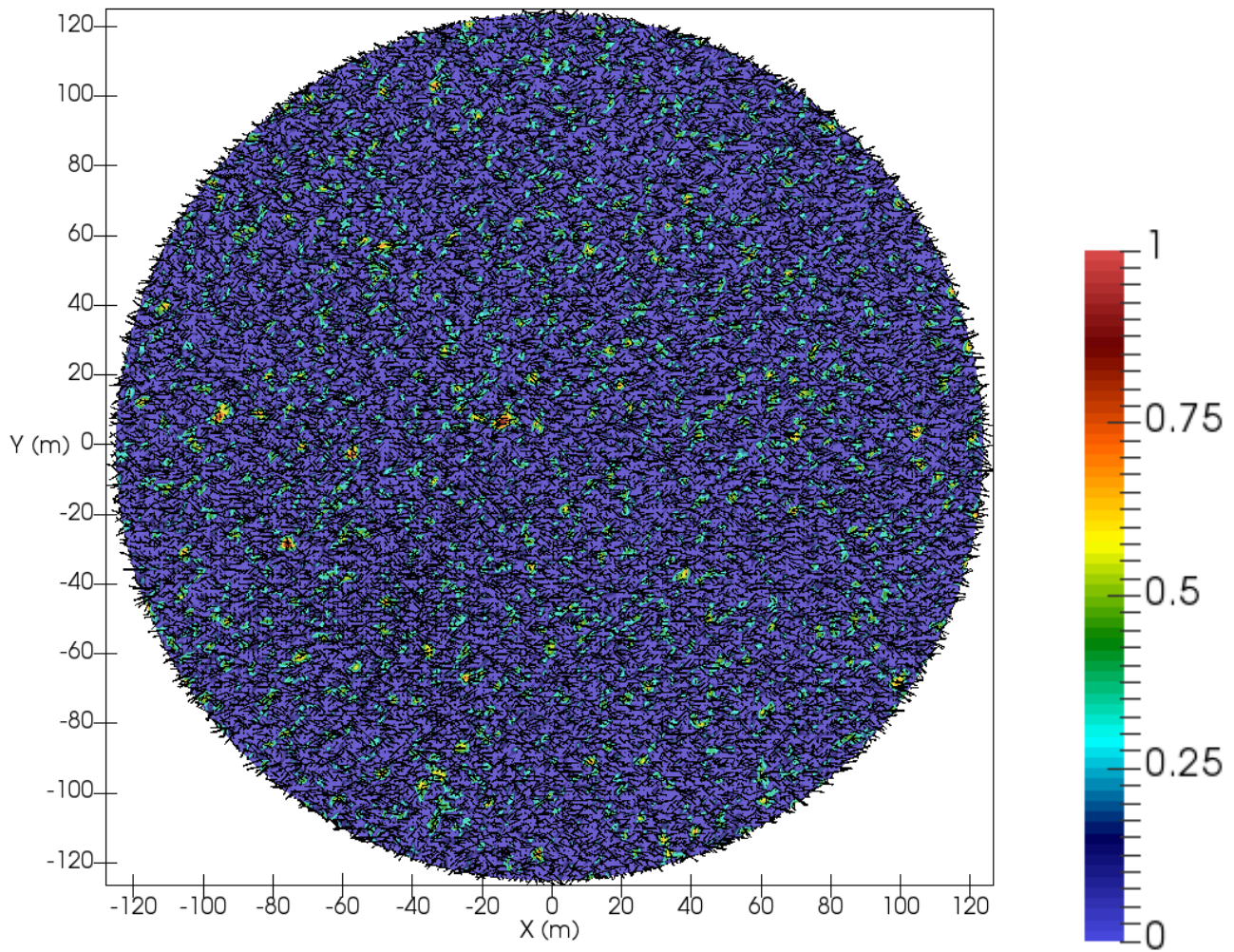

Figure 3.3: Landing pattern from Reiner Gamma simulation with ejected dust grains. 
range of 0.44 to $1.0 \mathrm{~m} / \mathrm{s}$. The initial orientation of each particle was randomized within the plane that lies tangential to the lunar surface at $\vec{r}_{i}$, and the initial angular velocity was randomly sampled from 0 to $2 \pi \mathrm{rad} / \mathrm{s}$ along each principle axis of the dust grain. This way, the rotation of each dust grain was tracked along a parabolic trajectory within $1.0 \mathrm{~m}$ of the lunar surface.

The simulation was allowed to run for over 12.5 million particles, and the results are shown in figure 3.4. The landing pattern, produced in the same fashion as the landing pattern in section 3.3 is superimposed on figures 3.4(a) and 3.4(b), The background in figure 3.4(a) is the local correlation plot, shown over the entire domain of the simulation. The background in figure 3.4(b) is the log of the magnetic field strength horizontal to the lunar surface, normalized with respect to the global maximum. Figure 3.4(c) shows the Reiner Gamma swirl, and is included for comparison to the simulated images.

The results of this simulation seem to indicate significant uniformity in grain orientation for regions with strong horizontal magnetic fields. These same regions correlate with the bright segments of the Reiner Gamma swirl, which is consistent with solar wind standoff. This leads the author to believe it may be reasonable to expect significant grain uniformity for the smallest fraction of lunar dust in regions of horizontal magnetic fields in the Reiner Gamma LMA. 


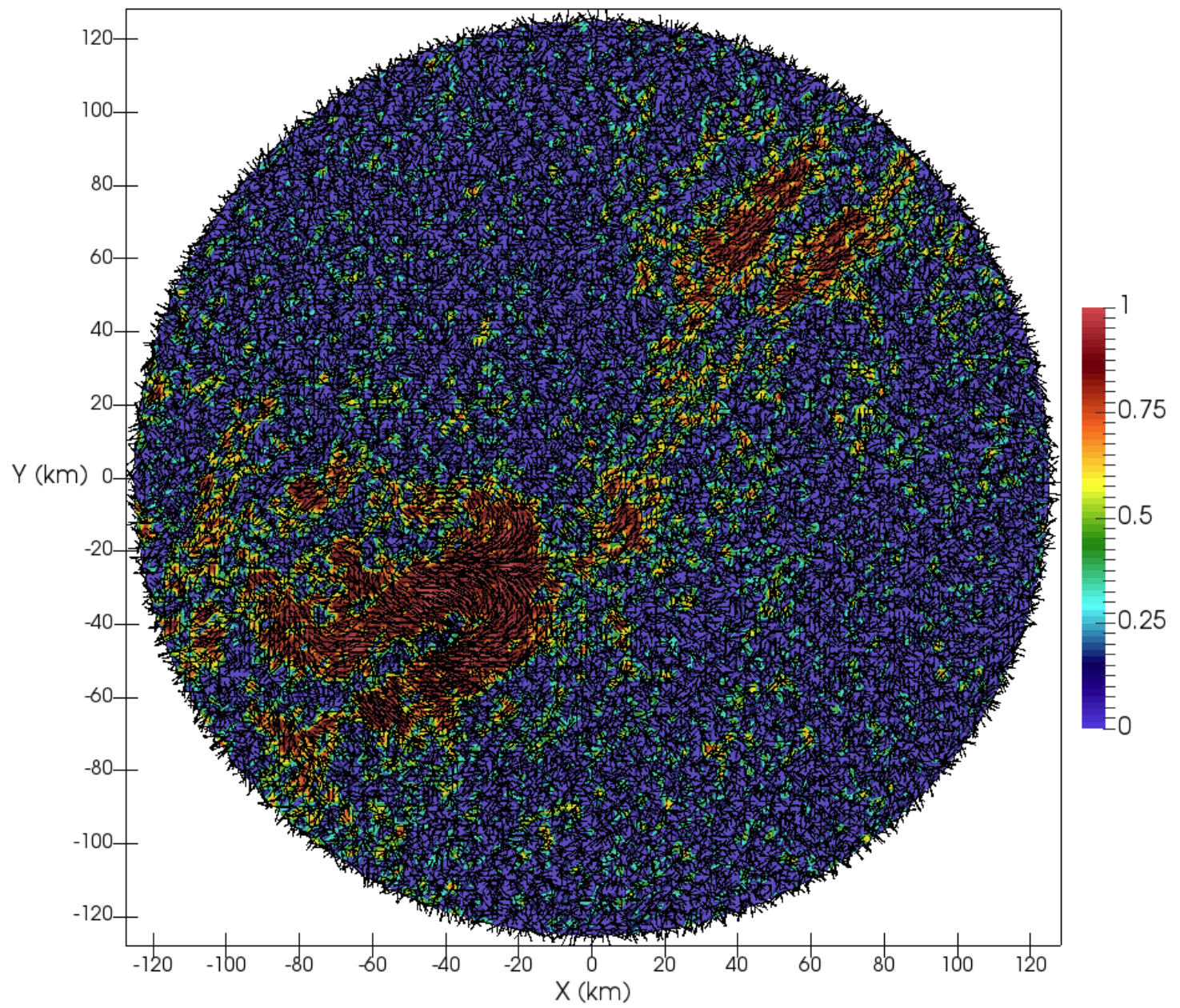

(a) Local corelation background

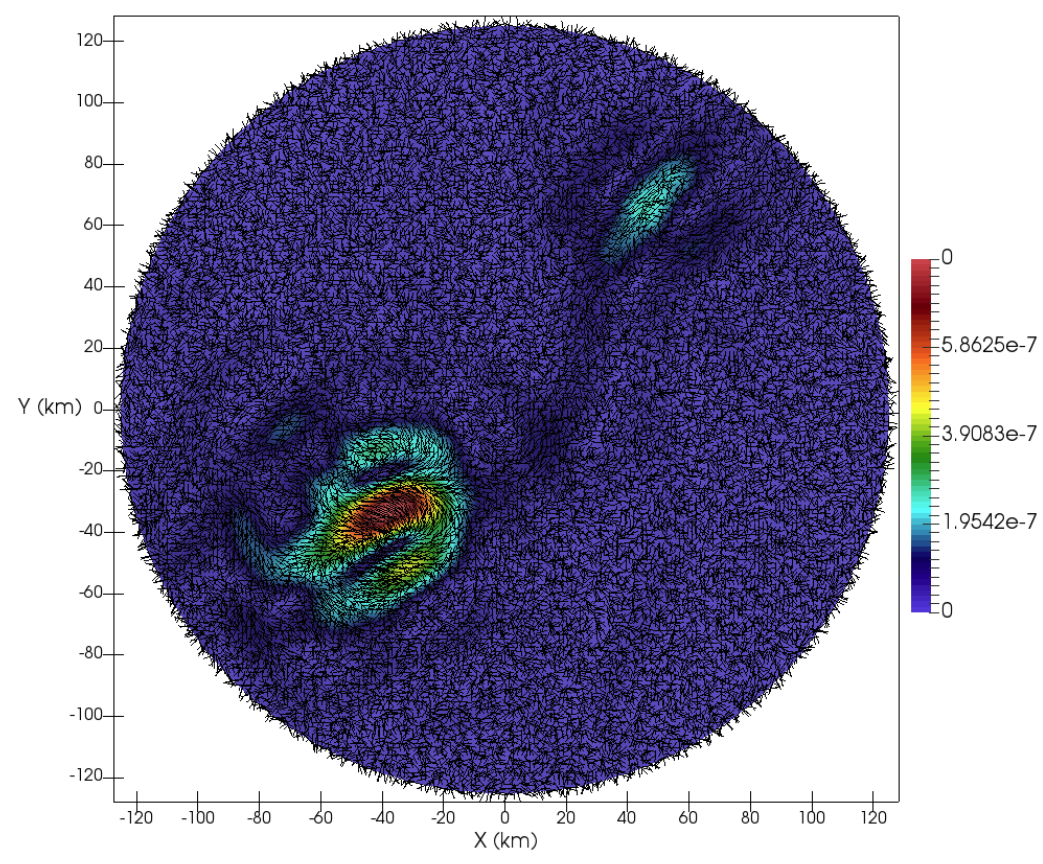

(b) Horizontal magnetic field background

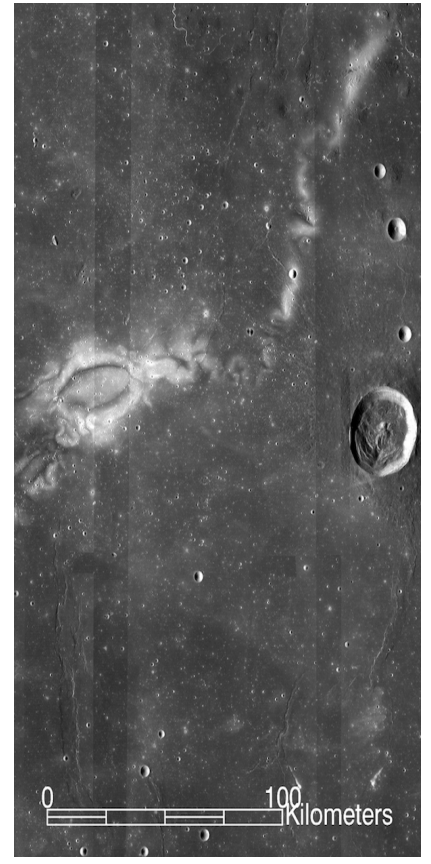

(c) Reiner Gamma swirl

Figure 3.4: Landing pattern from Reiner Gamma simulation with electrostatically lofted dust grains. 


\section{Chapter 4}

\section{Conclusion}

The unique photometric properties of lunar swirls suggest a reduced surface roughness when compared to similarly aged dust elsewhere in the lunar regolith. This anomaly has contributed significantly to the resiliency of the micrometeorite swarm theory, despite its inability to provide a satisfactory answer to the correlation between lunar swirls and LMAs. Similarly, the anomaly has contributed to the recent rise in popularity of the electrostatic transport model, while also hindering the ascendancy of solar wind standoff. Therefore, the resolution of this photometric anomaly is integral to the issue of swirl morphology.

Prior to the work presented in this thesis, there had been no consideration for the influence of individual dust rotations on the photometric properties of lunar swirls. Simulating these rotations, however, indicates that fine lunar grains $(<10 \mu \mathrm{m})$ may have a sufficiently high magnetic moment for emergent patterns to arise in the lunar regolith, especially in the vicinity of a strong magnetic field. This has been demonstrated for electrostatically lofted grains in the Reiner Gamma swirl, but not for particles ejected from a meteorite impact. This, however, does not indicate that ejected grains will not produce a similar landing pattern, since the failure to produce the pattern was most

likely the result of unrealistically low rotational energies compared to the linear kinetic energies of the simulated grains. Therefore, the orientation pattern produced in section 3.3 should serve as motivation for the continued exploration into the affects of dust rotation on swirl properties.

Considerable ground work has been laid in this regard, with numerous models attempting to explain the reflectance patterns observed from the lunar surface [42, [27], [40]. Should these 
models be adapted to demonstrate shallower phase functions for an anisotropic surface structure in a particulate media, then the photometric properties of swirls could possibly be explained with a dust rotation model that is compatible with solar wind standoff. This would open the door for novel laboratory experiments in which one could investigate how the reflectance properties of a magnetic and particulate media might change in the presence of a magnetic field. If such work should ever prove fruitful, then eventually the rotational energies necessary for significant grain alignment would have to be better constrained than what has been presented in this thesis. The work herein, however, does provide a starting point, as well as a first step in forming a foundational proof of concept for the dust rotation model. 


\section{Bibliography}

[1] David T Blewett, Ecaterina I Coman, B Hawke, Jeffrey J Gillis-Davis, Michael E Purucker, and Christopher G Hughes. Lunar swirls: Examining crustal magnetic anomalies and space weathering trends. Journal of Geophysical Research: Planets, 116(E2), 2011.

[2] Peter H Schultz, Leonard J Srnka, SI Pai, and S Menon. Cometary collisions on the moon and mercury. In Lunar and Planetary Science Conference, volume 11, pages 1009-1011, 1980.

[3] L. J. Srnka and P. H. Schultz. A Cometary Origin of Reiner-Gamma Magnetic Anomalies. In Lunar and Planetary Science Conference, volume 11 of Lunar and Planetary Science Conference, pages 1076-1078, March 1980.

[4] LL Hood and G Schubert. Lunar magnetic anomalies and surface optical properties. Science, 208(4439):49-51, 1980.

[5] LL Hood and CR Williams. The lunar swirls-distribution and possible origins. In Lunar and Planetary Science Conference Proceedings, volume 19, pages 99-113, 1989.

[6] Ian Garrick-Bethell, James W Head, and Carle M Pieters. Spectral properties, magnetic fields, and dust transport at lunar swirls. Icarus, 212(2):480-492, 2011.

[7] Paul Lucey, Randy L Korotev, Jeffrey J Gillis, Larry A Taylor, David Lawrence, Bruce A Campbell, Rick Elphic, Bill Feldman, Lon L Hood, Donald Hunten, et al. Understanding the lunar surface and space-moon interactions. Reviews in Mineralogy and Geochemistry, 60(1):83-219, 2006.

[8] Brett W Denevi, Mark S Robinson, Aaron K Boyd, David T Blewett, and Rachel L Klima. The distribution and extent of lunar swirls. Icarus, 273:53-67, 2016.

[9] Patrick C Pinet, Vladislav V Shevchenko, Serge D Chevrel, Yves Daydou, and Christine Rosemberg. Local and regional lunar regolith characteristics at reiner gamma formation: Optical and spectroscopic properties from clementine and earth-based data. Journal of Geophysical Research: Planets, 105(E4):9457-9475, 2000.

[10] Lon L. Hood. Lunar magnetic anomalies. 2014.

[11] Lon L. Hood. Central magnetic anomalies of nectarian-aged lunar impact basins: Probable evidence for an early core dynamo. Icarus, 211(2):1109 - 1128, 2011.

[12] D.L. Mitchell, J.S. Halekas, R.P. Lin, S. Frey, L.L. Hood, M.H. Acuña, and A. Binder. Global mapping of lunar crustal magnetic fields by lunar prospector. Icarus, 194(2):401 - 409, 2008. 
[13] Lon L. Hood and Natalia A. Artemieva. Antipodal effects of lunar basin-forming impacts: Initial 3d simulations and comparisons with observations. Icarus, 193(2):485 - 502, 2008. Saturn's Icy Satellites from Cassini.

[14] LL Hood and Z Huang. Formation of magnetic anomalies antipodal to lunar impact basins: Two-dimensional model calculations. Journal of Geophysical Research: Solid Earth, 96(B6):9837-9846, 1991.

[15] J. Gattacceca, M. Boustie, L. Hood, J.-P. Cuq-Lelandais, M. Fuller, N.S. Bezaeva, T. de Resseguier, and L. Berthe. Can the lunar crust be magnetized by shock: Experimental groundtruth. Earth and Planetary Science Letters, 299(1):42 - 53, 2010.

[16] Bruce Hapke. Space weathering from mercury to the asteroid belt. Journal of Geophysical Research: Planets, 106(E5):10039-10073, 2001.

[17] Sho Sasaki, Keiko Nakamura, Yoshimi Hamabe, Erika Kurahashi, and Takahiro Hiroi. Production of iron nanoparticles by laser irradiation in a simulation of lunar-like space weathering. Nature, 410(6828):555-557, 2001.

[18] John B. Adams and Robert L. Jones. Spectral reflectivity of lunar samples. Science, 167(3918):737-739, 1970.

[19] B. W. Hapke, A. J. Cohen, W. A. Cassidy, and E. N. Wells. Solar radiation effects on the optical properties of Apollo 11 samples. Geochimica et Cosmochimica Acta Supplement, 1:2199, 1970.

[20] R. V. Morris. Origins and size distribution of metallic iron particles in the lunar regolith. In S. A. Bedini, editor, Lunar and Planetary Science Conference Proceedings, volume 11 of Lunar and Planetary Science Conference Proceedings, pages 1697-1712, 1980.

[21] Richard V. Morris, Rex V. Gibbons, and Friedrich Hörz. Fmr thermomagnetic studies up to $900{ }^{\circ} \mathrm{c}$ of lunar soils and potential magnetic analogues. Geophysical Research Letters, 2(10):461-464, 1975.

[22] R. V. Morris. Surface exposure indices of lunar soils - A comparative FMR study. In D. C. Kinsler, editor, Lunar and Planetary Science Conference Proceedings, volume 7 of Lunar and Planetary Science Conference Proceedings, pages 315-335, April 1976.

[23] R. M. Housley, E. H. Cirlin, I. B. Goldberg, and H. Crowe. Ferromagnetic resonance studies of lunar core stratigraphy. In D. C. Kinsler, editor, Lunar and Planetary Science Conference $\underline{\text { Proceedings, }}$, volume 7 of Lunar and Planetary Science Conference Proceedings, pages 13-26, April 1976.

[24] G. K. Wehner. Sputtering effects on the moon's surface. ARS Journal, 31(3):438-439, 2017/10/07 1961.

[25] William Cassidy and Bruce Hapke. Effects of darkening processes on surfaces of airless bodies. Icarus, 25(3):371 - 383, 1975.

[26] Bruce Hapke, William Cassidy, and Edward Wells. Effects of vapor-phase deposition processes on the optical, chemical, and magnetic properties oe the lunar regolith. The moon, 13(1):339353, Mar 1975. 
[27] C. E. KenKnight, D. L. Rosenberg, and G. K. Wehner. Parameters of the optical properties of the lunar surface powder in relation to solar-wind bombardment. Journal of Geophysical Research, 72(12):3105-3129, 1967.

[28] D. L. Rosenberg and G. K. Wehner. Darkening of powdered basalt by simulated solar-wind bombardment. Journal of Geophysical Research, 69(15):3307-3308, 1964.

[29] Bruce Hapke. Darkening of silicate rock powders by solar wind sputtering. The moon, 7(3):342355, Sep 1973.

[30] Bruce Hapke. Effects of a simulated solar wind on the photometric properties of rocks and powders*. Annals of the New York Academy of Sciences, 123(2):711-721, 1965.

[31] L. Yin, T. Tsang, and I. Adler. On the ion-bombardment reduction mechanism. In D. C. Kinsler, editor, Lunar and Planetary Science Conference Proceedings, volume 7 of Lunar and Planetary Science Conference Proceedings, pages 891-900, April 1976.

[32] Lo I Yin, Subrata Ghose, and Isidore Adler. Investigation of a possible solar-wind darkening of the lunar surface by photoelectron spectroscopy. Journal of Geophysical Research, 77(8):13601367, 1972.

[33] L. Yin, T. Tsang, and I. Adler. ESCA studies on solar-wind reduction mechanisms. In Lunar and Planetary Science Conference Proceedings, volume 6 of Lunar and Planetary Science Conference Proceedings, pages 3277-3284, 1975.

[34] G. Strazzulla, E. Dotto, R. Binzel, R. Brunetto, M.A. Barucci, A. Blanco, and V. Orofino. Spectral alteration of the meteorite epinal (h5) induced by heavy ion irradiation: a simulation of space weathering effects on near-earth asteroids. Icarus, 174(1):31 - 35, 2005.

[35] Rosario Brunetto and Giovanni Strazzulla. Elastic collisions in ion irradiation experiments: A mechanism for space weathering of silicates. Icarus, 179(1):265 - 273, 2005.

[36] Maho Yamada, Sho Sasaki, Hiroko Nagahara, Akira Fujiwara, Sunao Hasegawa, Hajime Yano, Takahiro Hiroi, Hideo Ohashi, and Hisashi Otake. Simulation of space weathering of planetforming materials: Nanosecond pulse laser irradiation and proton implantation on olivine and pyroxene samples. Earth, planets and space, 51(11):1255-1265, 1999.

[37] LV Moroz, AV Fisenko, LF Semjonova, CM Pieters, and NN Korotaeva. Optical effects of regolith processes on s-asteroids as simulated by laser shots on ordinary chondrite and other mafic materials. Icarus, 122(2):366-382, 1996.

[38] R Brunetto, F Romano, A Blanco, S Fonti, M Martino, V Orofino, and C Verrienti. Space weathering of silicates simulated by nanosecond pulse uv excimer laser. Icarus, 180(2):546-554, 2006 .

[39] P. C. Pinet, A. Cord, S. Chevrel, and Y. Daydou. Optical Response and Surface Physical Properties of the Lunar Regolith at Reiner Gamma Formation from Clementine Orbital Photometry: Derivation of the Hapke Parameters at Local Scale. In S. Mackwell and E. Stansbery, editors, Lunar and Planetary Science Conference, volume 35 of Lunar and Planetary Science Conference, March 2004. 
[40] S. Chevrel, P. C. Pinet, A. Jehl, S. Besse, A. Cord, Y. Daydou, D. Baratoux, V. G. Kaydash, and Y. Shkuratov. Surface Physical Properties of the Lunar Regolith at Reiner Gamma: Characterization and Distribution Using Hapke Model Inversion. In S. Mackwell and E. Stansbery, editors, 37th Annual Lunar and Planetary Science Conference, volume 37 of Lunar and Planetary Science Conference, March 2006.

[41] C.D. Neish, D.T. Blewett, D.B.J. Bussey, S.J. Lawrence, M. Mechtley, and B.J. Thomson. The surficial nature of lunar swirls as revealed by the mini-rf instrument. Icarus, 215(1):186 - 196, 2011.

[42] Bruce Hapke. Bidirectional reflectance spectroscopy: 3. correction for macroscopic roughness. Icarus, 59(1):41 - 59, 1984.

[43] B. Hapke. Theory of Reflectance and Emittance Spectroscopy. Cambridge University Press, 2012.

[44] Yu G Shkuratov and NV Opanasenko. Polarimetric and photometric properties of the moon: Telescope observation and laboratory simulation: 2. the positive polarization. Icarus, 99(2):468-484, 1992.

[45] V Kaydash, M Kreslavsky, Yu Shkuratov, S Gerasimenko, P Pinet, J-L Josset, S Beauvivre, B Foing, et al. Photometric anomalies of the lunar surface studied with smart-1 amie data. Icarus, 202(2):393-413, 2009.

[46] MA Kreslavsky, Yu G Shkuratov, Yu I Velikodsky, VG Kaydash, DG Stankevich, and CM Pieters. Photometric properties of the lunar surface derived from clementine observations. Journal of Geophysical Research: Planets, 105(E8):20281-20295, 2000.

[47] M. A. Kreslavsky and Y. G. Shkuratov. Photometric anomalies of the lunar surface: Results from clementine data. Journal of Geophysical Research: Planets, 108(E3):n/a-n/a, 2003. 5015.

[48] Yu. Shkuratov, V. Kaydash, S. Gerasimenko, N. Opanasenko, Yu. Velikodsky, V. Korokhin, G. Videen, and C. Pieters. Probable swirls detected as photometric anomalies in oceanus procellarum. Icarus, 208(1):20 - 30, 2010.

[49] Yurij Shkuratov, Larissa Starukhina, Harald Hoffmann, and Gabriele Arnold. A model of spectral albedo of particulate surfaces: Implications for optical properties of the moon. Icarus, 137(2):235 - 246, 1999.

[50] Larissa V Starukhina and Yuriy G Shkuratov. Swirls on the moon and mercury: Meteoroid swarm encounters as a formation mechanism. Icarus, 167(1):136-147, 2004.

[51] J. F. Bell and B. R. Hawke. Recent comet impacts on the moon - the evidence from remotesensing studies. Publications of the Astronomical Society of the Pacific, 99(618):862, 1987.

[52] Yuriy G Shkuratov, Dmitriy G Stankevich, Vadim G Kaydash, Vitaliy V Omelchenko, Carle M Pieters, Patrick C Pinet, Serge D Chevrel, Yves H Daydou, Bernard H Foing, Zoran Sodnik, et al. Composition of the lunar surface as will be seen from smart-1: A simulation using clementine data. Journal of Geophysical Research: Planets, 108(E4), 2003.

[53] Thomas Gold and Steven Soter. Cometary impact and the magnetization of the moon. Planetary and Space Science, 24(1):45 - 54, 1976. 
[54] R.P. Lin, K.A. Anderson, and L.L. Hood. Lunar surface magnetic field concentrations antipodal to young large impact basins. Icarus, 74(3):529 - 541, 1988.

[55] N. C. Richmond, L. L. Hood, D. L. Mitchell, R. P. Lin, M. H. Acuña, and A. B. Binder. Correlations between magnetic anomalies and surface geology antipodal to lunar impact basins. Journal of Geophysical Research: Planets, 110(E5):n/a-n/a, 2005. E05011.

[56] Joseph B. Nicholas, Michael E. Purucker, and Terence J. Sabaka. Age spot or youthful marking: Origin of reiner gamma. Geophysical Research Letters, 34(2):n/a-n/a, 2007. L02205.

[57] Doug Hemingway and Ian Garrick-Bethell. Magnetic field direction and lunar swirl morphology: Insights from airy and reiner gamma. Journal of Geophysical Research: Planets, 117(E10), 2012.

[58] N. C. Richmond and L. L. Hood. A preliminary global map of the vector lunar crustal magnetic field based on lunar prospector magnetometer data. Journal of Geophysical Research: Planets, 113(E2):n/a-n/a, 2008. E02010.

[59] J. E. McCoy and D. R. Criswell. Evidence for a high altitude distribution of lunar dust. In Lunar and Planetary Science Conference Proceedings, volume 5 of Lunar and Planetary Science Conference Proceedings, pages 2991-3005, 1974.

[60] David R Criswell. Horizon-glow and the motion of lunar dust. In Photon and Particle Interactions with Surfaces in Space, pages 545-556. Springer, 1973.

[61] MM Abbas, D Tankosic, PD Craven, JF Spann, A LeClair, and EA West. Lunar dust charging by photoelectric emissions. Planetary and Space Science, 55(7):953-965, 2007.

[62] X Wang, J Schwan, H-W Hsu, E Grün, and M Horányi. Dust charging and transport on airless planetary bodies. Geophysical Research Letters, 43(12):6103-6110, 2016.

[63] Herbert A. Zook and James E. McCoy. Large scale lunar horizon glow and a high altitude lunar dust exosphere. Geophysical Research Letters, 18(11):2117-2120, 1991.

[64] Timothy J. Stubbs, Richard R. Vondrak, and William M. Farrell. A dynamic fountain model for lunar dust. Advances in Space Research, 37(1):59 - 66, 2006. The Moon and Near-Earth Objects.

[65] M. Neugebauer, C.W. Snyder, D.R. Clay, and B.E. Goldstein. Solar wind observations on the lunar surface with the apollo-12 alsep. Planetary and Space Science, 20(10):1577 - 1591, 1972.

[66] K. Takahashi, T. Kaneko, and R. Hatakeyama. Double layer created by electron cyclotron resonance heating in an inhomogeneously magnetized plasma with high-speed ion flow. Physics of Plasmas, 15(7):072108, 2008.

[67] Jan Deca, Andrey Divin, Bertrand Lembège, Mihály Horányi, Stefano Markidis, and Giovanni Lapenta. General mechanism and dynamics of the solar wind interaction with lunar magnetic anomalies from 3-d particle-in-cell simulations. Journal of Geophysical Research: Space Physics, 120(8):6443-6463, 2015.

[68] Timothy D Glotch, Joshua L Bandfield, Paul G Lucey, Paul O Hayne, Benjamin T Greenhagen, Jessica A Arnold, Rebecca R Ghent, and David A Paige. Formation of lunar swirls by magnetic field standoff of the solar wind. Nature communications, 6, 2015. 
[69] Fun-Dow Tsay, Sunney I Chan, and Stanley L Manatt. Ferromagnetic resonance of lunar samples. Geochimica et Cosmochimica Acta, 35(9):865-875, 1971.

[70] DW Strangway, EE Larson, and GW Pearce. Magnetic studies of lunar samples-breccia and fines. Geochimica et Cosmochimica Acta Supplement, 1:2435, 1970.

[71] Grant Heiken, David Vaniman, and Bevan M French. Lunar sourcebook: A user's guide to the Moon. CUP Archive, 1991.

[72] Jack B Kuipers et al. Quaternions and rotation sequences, volume 66. Princeton university press Princeton, 1999.

[73] Hideo Tsunakawa, Futoshi Takahashi, Hisayoshi Shimizu, Hidetoshi Shibuya, and Masaki Matsushima. Surface vector mapping of magnetic anomalies over the moon using kaguya and lunar prospector observations. Journal of Geophysical Research: Planets, 120(6):1160-1185, 2015. 2014JE004785.

[74] J. Deca, A. Divin, X. Wang, B. Lembège, S. Markidis, G. Lapenta, and M. Horányi. Solar Wind Interaction with Lunar Magnetic Anomalies: Reiner Gamma. In EGU General Assembly Conference Abstracts, volume 18 of EGU General Assembly Conference Abstracts, pages EPSC2016-3146, April 2016.

[75] Jamey R. Szalay and Mihály Horányi. Lunar meteoritic gardening rate derived from in situ ladee/ldex measurements. Geophysical Research Letters, 43(10):4893-4898, 2016. 2016GL069148. 


\section{Appendix A}

\section{Quaternion Parameterization}

\section{A.1 Introduction to Quaternions}

All statements concerning quaternions are referenced to [72] and the citations therein. Quaternions are a four dimensional number structure forming a closed non-commutative division algebra. They are frequently used in navigation systems and computer animation because of their stability when modeling highly rotationally dynamic systems. Each quaternion has one real and three imaginary components, which relate to one another by the following rules.

$$
\begin{gathered}
q=q_{0}+q_{1} \mathbf{i}+q_{2} \mathbf{j}+q_{3} \mathbf{k} \\
\mathbf{i j}=\mathbf{k}, \quad \mathbf{j} \mathbf{i}=-\mathbf{k} \\
\mathbf{j} \mathbf{k}=\mathbf{i}, \quad \mathbf{k} \mathbf{j}=-\mathbf{i} \\
\mathbf{k} \mathbf{i}=\mathbf{j}, \quad \mathbf{i k}=-\mathbf{j}
\end{gathered}
$$

Any three dimensional vector is therefore a subset of the quaternion group, and is considered to be a pure quaternion, which is a quaternion with purely imaginary components. By convention, $\mathbf{i}$, $\mathbf{j}$ and $\mathbf{k}$ corresponding to $\hat{x}, \hat{y}$ and $\hat{z}$, respectively. Therefore, quaternions allow for the multiplication and division of vectors by allowing a fourth degree of freedom, which is the real component of a quaternion. The process of quaternion division is performed using a complex conjugate, similar to that of a complex number. Therefore, the conjugate of equation A.1 is equation A.2, and their 
product is equation A.3.

$$
\begin{aligned}
q^{*} & =q_{0}-q_{1} \mathbf{i}-q_{2} \mathbf{j}-q_{3} \mathbf{k} \\
q q^{*} & =\sqrt{q_{0}^{2}+q_{1}^{2}+q_{2}^{2}+q_{3}^{2}}
\end{aligned}
$$

Any unit quaternion, where $q q^{*}=1$, can be described with a unit vector $\hat{u}$ and an angle $\alpha$ using equation A.4. The geometric interpretation of such an object says that a unit quaternion will describe a rotation of $\alpha$ about the unit vector $\hat{u}$.

$$
\begin{aligned}
& q=\cos \left(\frac{\alpha}{2}\right)+\hat{u} \sin \left(\frac{\alpha}{2}\right) \\
& \hat{u}=u_{x} \mathbf{i}+u_{y} \mathbf{j}+u_{z} \mathbf{k}
\end{aligned}
$$

Such a rotation can be applied to any arbitrary vector $\vec{v}$, transforming $\vec{v} \rightarrow \tilde{\vec{v}}$ by equation A.6 or $\tilde{\vec{v}} \rightarrow \vec{v}$ by equation A.7.

$$
\begin{aligned}
& \tilde{\vec{v}}=q \vec{v} q^{*} \\
& \vec{v}=q^{*} \tilde{\vec{v}} q
\end{aligned}
$$

It can be shown that Euler's formula for complex numbers can be extended to quaternions, so that any unit quaternion can be described by equation A.8.

$$
q=\operatorname{Exp}\left[\left(\frac{\alpha}{2}\right) \hat{u}\right]
$$

If one then takes the first time derivative of the natural logarithm of equation A.8, they will get equation A.9.

$$
\frac{\dot{q}}{q}=\frac{1}{2} \frac{d}{d t}(\alpha \hat{u})
$$

Since $\hat{u}$ can be interpreted geometrically as an axis of rotation, and $\alpha$ as the angle of rotation about that axis, then the time derivative of $\alpha \hat{u}$ is, by definition, an angular velocity. Therefore, 
equation A.9 can be simplified to A.10, which shows how the angular velocity $\vec{\omega}$ of a rigid body can be parameterized using quaternions.

$$
\vec{\omega}=2 \dot{q} q^{*}
$$

\section{A.2 Application of the Quaternion Number Structure}

This structure provides a stable configuration for any rotational system, making it superior to Euler angles. In order to apply these relations to the simulation, I define the body frame by equations A.11, A.12 and A.13.

$$
\begin{aligned}
& \hat{e}_{1}^{\prime}=\mathbf{i} \\
& \hat{e}_{2}^{\prime}=\mathbf{j} \\
& \hat{e}_{3}^{\prime}=\mathbf{k}
\end{aligned}
$$

In a simulation, when each dust grain is initialized, its orientation is randomized with an initial quaternion $q$, which is constrained by the specific conditions of the simulation and then calculated using equation A.4. Any vector in the body frame can then be transformed to the space frame using equation A.14, and a transformation from the space frame to the body frame can be carried out by the inverse operation. Similarly, with an angular velocity initialized in the body frame $\vec{\omega}^{\prime}$, the first time derivate of $q$ can be determine from equation A.15.

$$
\begin{gathered}
\hat{e}_{n}=q \hat{e}_{n}^{\prime} q^{*} \\
\dot{q}=\frac{1}{2} q \vec{\omega}^{\prime}
\end{gathered}
$$

One can use equation A.15 to find the second time derivative of the system's quaternion, simplifying to equation A.17. Then, using Euler's formula for $\dot{\vec{\omega}}^{\prime}$ as described in equation 2.2 , equation A.17 becomes an integrable equation for $q$, thus characterizing the orientation of the 
grain along its entire trajectory.

$$
\begin{aligned}
& \ddot{q}=\frac{1}{2} \dot{q} \vec{\omega}^{\prime}+\frac{1}{2} q \dot{\vec{\omega}}^{\prime} \\
& \ddot{q}=\dot{q} q^{*} \dot{q}+\frac{1}{2} q \dot{\vec{\omega}}^{\prime}
\end{aligned}
$$

